\title{
The anatomy of a tornillo: puzzles from three-component measurements at Galeras volcano (Colombia)
}

\author{
Dieter Seidl $\left({ }^{1}\right)$, Margaret Hellweg $\left({ }^{2}\right)$, Horst Rademacher $\left({ }^{3}\right)$, \\ Diego M. Gómez M. $\left({ }^{4}\right)$ and Roberto A. Torres C. $\left({ }^{4}\right)$ \\ (1) Bundesanstalt für Geowissenschaften und Rohstoffe, Seismologisches Zentralobservatorium Gräfenberg, \\ Erlangen, Germany \\ $\left.{ }^{2}\right)$ Institut für Geophysik, Universität Stuttgart, Germany \\ $\left(^{3}\right)$ Geo Enterprises Orinda, CA, U.S.A. \\ $\left(^{4}\right)$ INGEOMINAS - Observatorio Vulcanológico y Sismológico, Pasto, Colombia
}

\begin{abstract}
Many of the recent ash eruptions of Galeras volcano (Colombia) have been preceded by tornillos. These unusual seismic events of unknown origin have screw-like profiles on seismograms and can last up to several minutes. In March, 1996, a broadband, three-component seismometer station was installed $1.6 \mathrm{~km}$ south of Cono, Galeras' active vent. With high quality recordings acquired by this system, and methods which work well with narrow band signals, we investigate the frequency content and polarization properties of two tornillos. They have slighty different predominant frequencies, $3.0420 \mathrm{~Hz}$ and $3.0595 \mathrm{~Hz}$ which remain highly stable for the entire tornillo duration. The polarization analysis shows that the polarization for the two events also differs slightly but remains constant. Most of their energy is linearly polarized and lies in the horizontal plane. If these waves are taken to be $\mathrm{SH}$ waves, the remaining energy is confined to the beginning of the tornillo during its initiation or excitation. The remainder of the tornillo is characterized by exponential decay. These observations provide parameters for the classification of tornillos and constraints for source models.
\end{abstract}

Key words Galeras - tornillo - polarization analytic function - parametrization

\section{Introduction}

A fundamental difference exists between tectonic earthquakes and some classes of seismic events associated with volcanic activity. The excitation process for tectonic earthquakes is -

Mailing address: Dr. Dieter Seidl, Seismologisches Zentralobservatorium, Mozartstr. 57, D-91052 Erlangen, Germany; e-mail: seidl@szgrf.uni-erlangen.de except perhaps for some very deep focus events always impulsive, i.e. a brittle fracture of solid rock due to a high accumulation of tectonic stress. These types of earthquakes exist in a volcanic regime as well, either as extensional fractures due to inflation of the volcanic edifice or as shear fractures along preexisting faults. There are however seismic signals associated with magmatic activity, which are not generated by impulsive excitation. Volcanic tremor is a classical example for such signals. It is most likely generated by repetitive, stationary processes in the liquid and gaseous phases of the volcano. Resonances in the fluid add to the «non-impulsiveness» of the signals (see Mar- 
tinelli, 1991, for a review). Long term seismic observations at several volcanoes located at convergent plate boundaries have revealed another class of relatively rare seismovolcanic events, called tornillos (Torres et al., 1996; Gómez and Torres, 1997; Gómez et al., 1999). At first glance they appear to be a combination of an impulsive excitation and a resonance within the volcano, because on seismograms these tornillos have an abrupt beginning followed by a long, resonant vibration which may last for several minutes.

Until recently, most tornillo-events were recorded with short period vertical seismometers. These measurements reveal the tornillos' unique, monochromatic spectral character. They do not, however, permit a thorough investigation of the possible excitation and resonance parameters. In March 1996 we deployed a three-component, digital broadband seismic recording station approximately $1.6 \mathrm{~km}$ south of the active vent of Galeras volcano in Southern Colombia, the «type location» for tornillo-events (see Gómez et al., 1999 for a detailed description). Unfortunately, the volcano fell silent shortly after the instrument deployment. Nevertheless, in April 1996 we recorded five tornillos, the two strongest of which are the focus of the investigation presented in this paper.

In tornillo research, digital recordings from three-component broadband seismometers have several advantages over short period analogue recordings with vertical instruments. With a three-component seismometer, the polarization of the seismic waves associated with a tornillo can be measured precisely as a function of time. In addition, digital recordings with high dynamic range and high resolution permit a detailed analysis of its spectral parameters and amplitude. In this paper, we present results from the analysis of the polarization and the spectral characteristics of the recordings of two tornillos. We will also model its amplitude behaviour. This enables us to describe the anatomy of tornillos and their possible origin with measurable parameters. More general conclusions about the mechanism and the location of the source are not possible, however, until more tornillos have been recorded on a network of three-component, high dynamic range stations.

\section{Data}

The station, located at Crater-HQ (for a map, see Gómez et al., 1999), is equipped with a broadband seismometer STS-2 (Wielandt and Streckeisen, 1982) and a PDAS-Datalogger (Teledyne Brown Engineering, Geotech Instruments) with continuous recording at $50 \mathrm{~Hz}$ on a 540 Mbyte hard disk. The digital sensitivity was 2 $\mathrm{nm} / \mathrm{s}$ in the Least Significant Bit (LSB). Figure 1 shows the three component seismograms for two tornillos, $\mathrm{T} 1$ and $\mathrm{T} 2$, recorded within $5 \mathrm{~h}$ of each other (for other examples of tornillos, see Gómez et al., 1999). In addition to the tornillos, the broadband recordings contain longer period ocean microseisms and other high frequency signals. To improve the signal-to-noise ratio for the analysis of the tornillo, the broadband data were therefore prefiltered using a Butterworth band pass filter (2-4 Hz) of order 6 . The seismic energy for both events is concentrated in $\mathrm{N}$ and E components.

\section{Theoretical considerations}

To all appearances a tornillo is a monochromatic signal with changing amplitude (Torres et al., 1996; Gómez and Torres, 1997; Gómez et al., 1999). The precision of frequency measurements made using spectra, however, depend on the bandwidth of the signal, which may be influenced by frequency variations. Thus, the most general description of a tornillo, $s(t)$, allows variations in the frequency and phase as well as the amplitude

$$
s(t)=a(t) \sin (\omega(t) t+\varphi(t)) \vec{n}(t)
$$

where $\vec{n}(t)$ is the polarization, $a(t)$ the amplitude, $\omega(t)$ the frequency und $\varphi(t)$ the phase. Each of these precisely measurable parameters contains important information about the source, its excitation and its resonant behaviour. For example, the polarization of the seismic wave field generated by a non-moving, stationary source process would not change at any given location over time. A detailed investigation of the spectral and phase variations during the tor- 

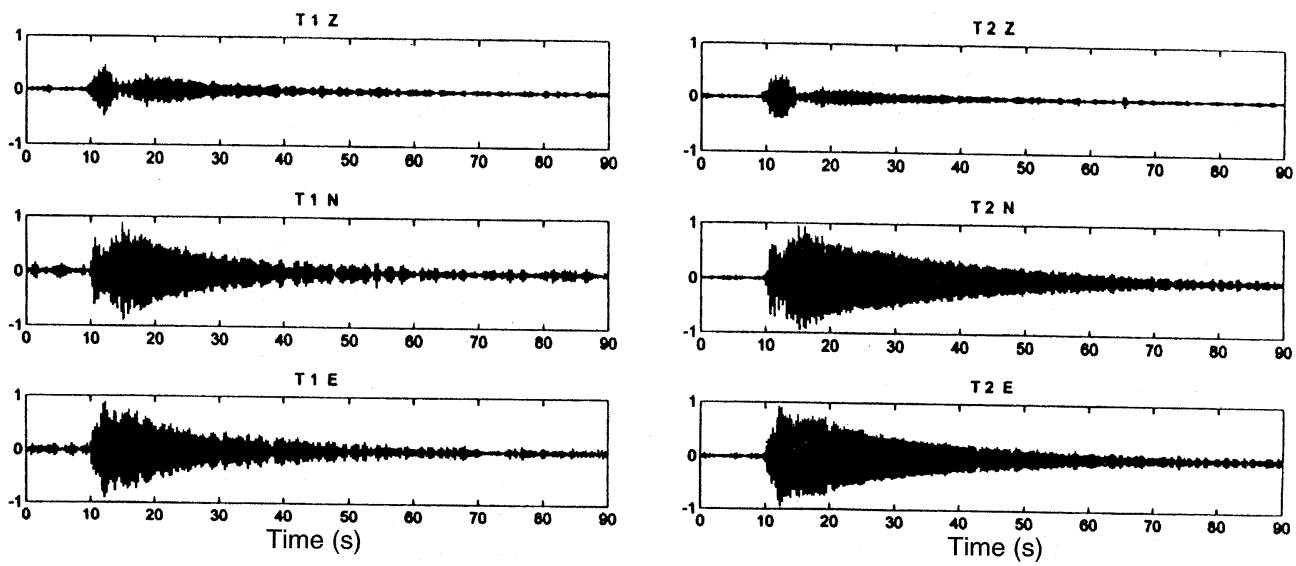

Fig. 1. Three-component velocity seismograms: bandpass filtered broadband velocity seismograms (units: $\mu \mathrm{m} / \mathrm{s}$ ) for two tornillos T1 and T2 recorded with a STS-2 seismometer at crater station. Z, N, E: vertical, northSouth, east-west component. Time at $t=0:$ T1: 9. April 1996, 20:31:34 UTC; T2: 10. April 1996 01:34:11 UTC. Bandpass filter: Butterworth: 6th order, frequency band $(2.0,4.0) \mathrm{Hz}$.

nillo can render additional information about the source and the oscillatory resonance mechanism in the fluid phase of Galeras.

\section{Polarization}

In the investigation of tectonic earthquakes, polarization analysis is used to discriminate between different wave types and to locate the source (Kanasewich, 1981; Plešinger et al., 1986). This technique cannot be applied to the investigation of the tornillo wavefield at Galeras without additional assumptions. With only one station we cannot determine the wave type and direction towards the source independently. Nevertheless, polarization analysis can yield important information about the wave field and therefore about the anatomy of a tornillo. Figure 2 shows a perspective view of the threedimensional particle motion diagram for a $5 \mathrm{~s}$ time window near the maximum of the seismogram of T2. Visual inspection shows that the particle motion in this segment is almost completely horizontal and in a SW-NE direction.

To calculate the inclination angle and azimuth, we apply a least-square-fit to the polarization ellipsoid. Its principle axes system $X_{1}-X_{2}$ -
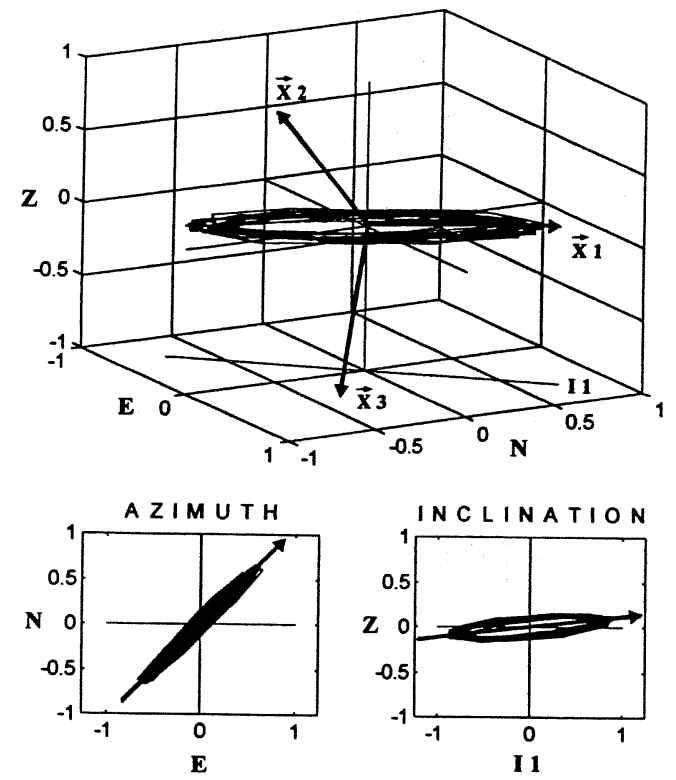

Fig. 2. Particle motion diagrams: 3D particle motion plot for a $5 \mathrm{~s}$ segment of T2 showing almost horizontal polarization. $X_{1}-X_{2}-X_{3}$ are the eigenvectors of the covariance matrix. The square root ratio of the eigenvalues is about 5:1:1. $X_{2}$ and $X_{3}$ are zoomed by 8 for visual clearness. The $2 \mathrm{D}$ plots indicate the azimuth and inclination of the major axis $X_{1}$. 
$X_{3}$ is defined by the eigenvalues $\left(\lambda_{1}, \lambda_{2}, \lambda_{3}\right)$ and the eigenvectors $\left(\vec{e}_{1}, \vec{e}_{2}, \vec{e}_{3}\right)$ of the $3 \times 3$ covariance matrix of the three-component seismograms of $\mathrm{T} 2$ in fig. 1, with

$$
\vec{X}_{i}=\lambda_{i} \vec{e}_{i}
$$

where $i=1,2,3$ for the major, intermediate and minor eigenvalues (Seidl and Hellweg, 1991).
In fig. 3 the two tornillos have been rotated into their respective eigensystems. Note the similarity between their components. In the eigenvalue system, both tornillos can be resolved into three very different signals. $X_{1}$ is very simple signal with a rapid increase in amplitude followed by a long decay. The seismogram for $X_{2}$ is a single, brief pulse, while $X_{3}$ is a longer, low amplitude pulse.
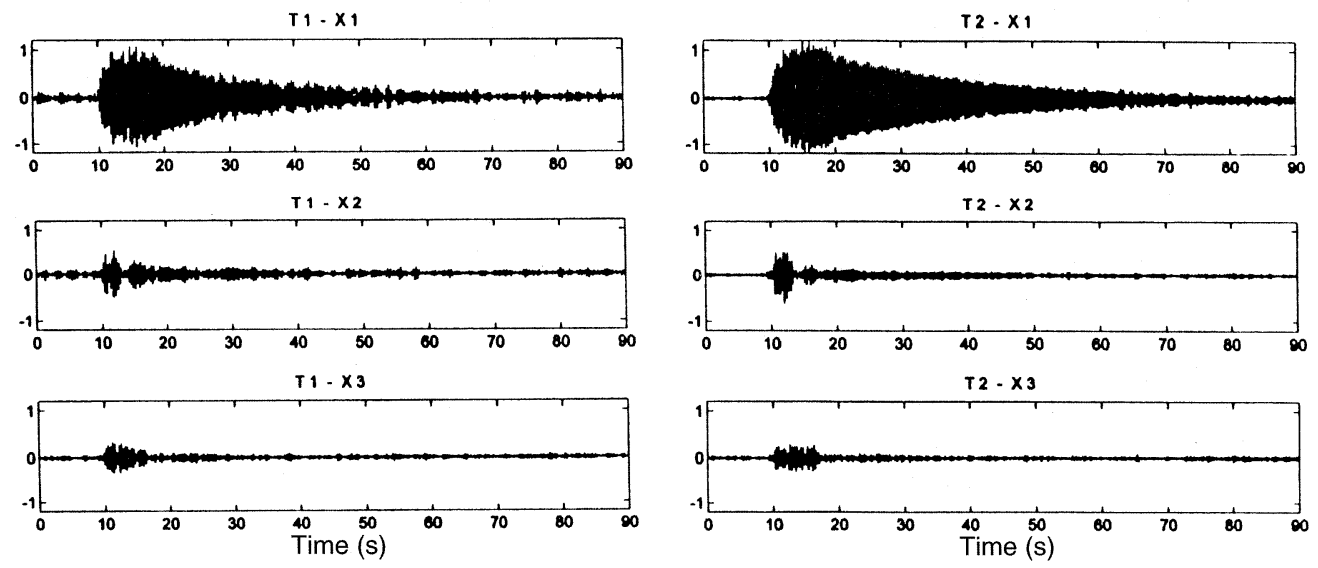

Fig. 3. Eigensystem seismograms: transformation of the seismograms in fig. 1 into the eigensystem $X_{1}-X_{2}-X_{3}$ of the covariance matrix from fig. 2.
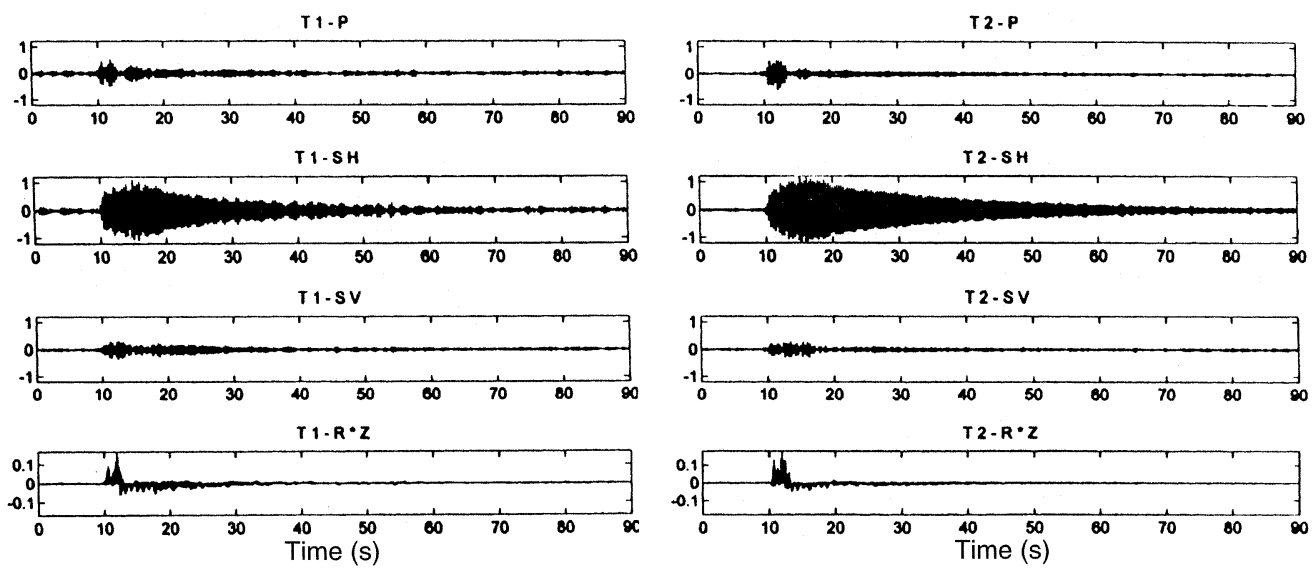

Fig. 4. $P-S H-S V$ seismograms: transformation of the seismogram in fig. 1 into a $P-S H-S V$ system with the $X_{2}$-axis in fig. 2 taken to be the longitudinal $P$-direction. The mean values for azimuth (against north) and inclination (against Z) for the $X_{2}$-axis are $a z_{1}=142^{\circ}$ and $i n_{1}=66^{\circ}$ for $\mathrm{T} 1$ and $a z_{2}=139^{\circ}$ and $i n_{2}=47^{\circ}$ for $\mathrm{T} 2$, respectively. 
Along with the azimuth and inclination of the major axis of the polarization ellipsoid, the rectilinearity can be used as a characteristic for the parametrization and classification of tornillo three-component seismograms. The degree to which the polarization is linear can be described by the rectilinearity function (Kanasewich, 1981)

$$
F=1-\lambda_{2} / \lambda_{1}
$$

$(F=1$ : perfectly linear polarization, $F=0$ : unpolarized). The values for $\mathrm{T} 1$ and $\mathrm{T} 2$ are $F_{1}=0.93$ and $F_{2}=0.95$, indicating a high degree of rectilinearity for both tornillos. The direction of polarization is given by the azimuth, $a z$, and inclination, in, of the eigenvector for the major eigenvalue. The azimuth and inclination particle motion diagrams in fig. 2 show a mean azimuth of $a z_{2}=44^{\circ}$ east of north and a mean inclination of $i n_{2}=84^{\circ}$ from vertical, which confirm the observation that most of the seismic energy is concentrated in the horizontal plane.

In earthquake seismology, such a clear and time invariant polarization would indicate the direction to the source. Similar logic cannot be applied to the tornillo recordings at Galeras, because we have no independent means to determine which type of waves make up the tornillo wavefield. On the other hand, we can determine a probable wave coordinate system from assumptions about the relative polarizations of $P, S V$ and $S H$ waves. $S H$ waves are, in general, horizontally polarized, while the polarizations of $P$ and $S V$ waves share a vertical plane. Thus, it is intuitive to designate $X_{1}$ as the $S H$ direction. In this case, particle motion due to $P$ and $S V$ waves must lie in the $X_{2}-X_{3}$ plane. If we assign $X_{2}$ as the $P$ direction, the resulting seismograms are shown in fig. 4. Both tornillos then begin with a single, brief wavegroup of $P$-energy which is superimposed on the $\mathrm{SH}$-motion. This wavegroup can be seen in greater detail in fig. 5 . In the chosen wave system, the product of the radial with the vertical component, $R \boldsymbol{Z}$, is positive, the typical discriminant for $P$-wave polarization. Note the similarity of the fine structure in the signal form between the two tornillos, especially the double-pulse pattern revealed in radial seismogram and in the $\boldsymbol{R Z}$ trace.
T 1 - P

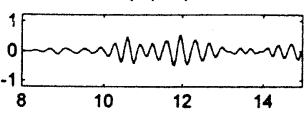

T $1 \cdot S \mathrm{H}$

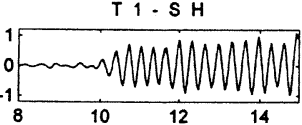

T 1 - R

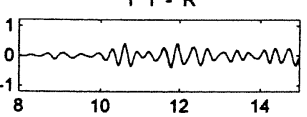

$T 1 \cdot R \cdot Z$
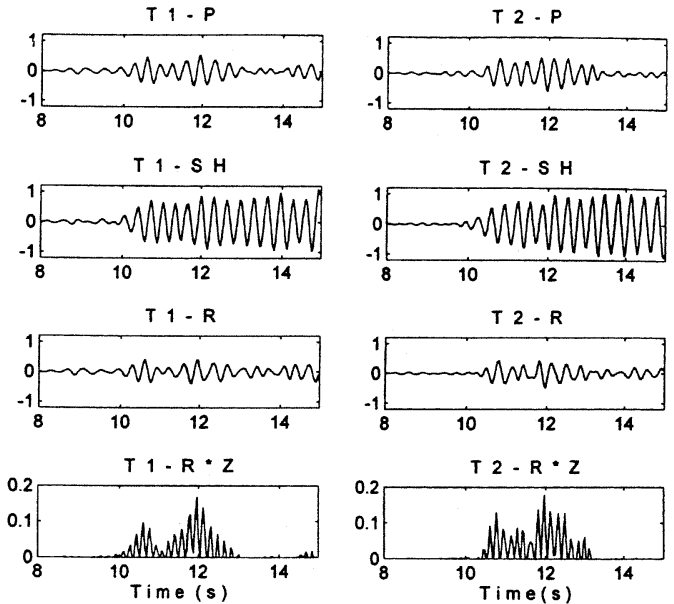

Fig. 5. P-wave transient: seismograms for $P, S H, R$ (radial) and $\mathrm{R} * \mathrm{Z}$ (rectilinear motion) for the beginning of T1 and T2 in fig. 4 showing the $P$-wavegroup (duration 3 and $4 \mathrm{~s}$ for $\mathrm{T} 1$ and $\mathrm{T} 2$, respectively) with a double pulse pattern superimposed on the $S H$-motion which is much stronger.

The eigensystem in fig. 3 is a mean coordinate system estimated from the covariance matrix for the complete tornillo seismogram. If the mean polarization is different for tornillos with the same spectral characteristics, it is probably due to variations in the time or spatial averages of the tornillos' locations or radiation patterns. On the other hand, changes in the instantaneous polarization for a single tornillo, measured from sequential intervals, may indicate movement of the source or a change in radiation pattern.

Figure 6 shows the time variations of the azimuth and inclination of the major axis of the polarization ellipsoid in fig. 2 for both tornillos. It has been estimated for moving $5 \mathrm{~s}$ intervals. Within the standard deviations, the azimuths for the two tornillos are the same. Their inclination angles, however, differ by about $10^{\circ}$. The variations of azimuth and inclination as a function of the time are similar for $\mathrm{T} 1$ and $\mathrm{T} 2$ with a strong deviation during the onset segment caused by the transient signal shown in fig. 5 . If the source is near the active crater, these variations in the azimuth and inclination imply that its linear extension cannot exceed $100 \mathrm{~m}$. 
T 1
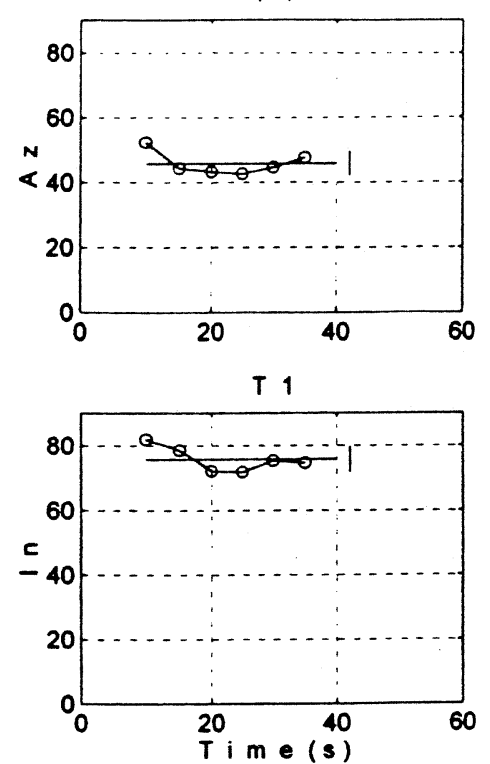

T 2
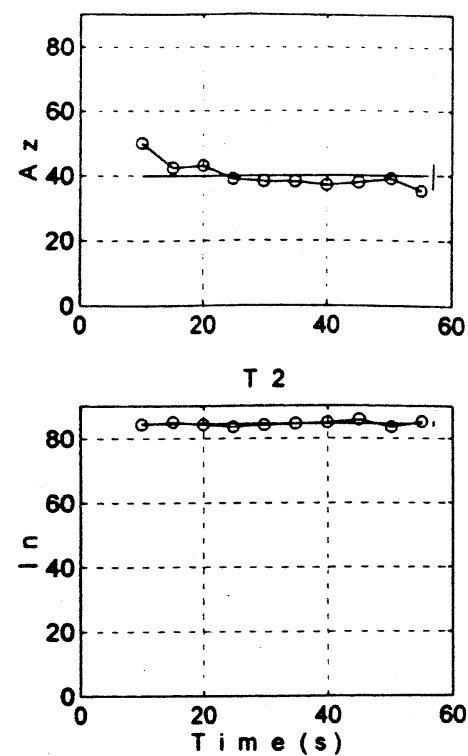

Fig. 6. Time variation of polarization: time variation of azimuth $(A z)$ and inclination $(I n)$ of eigenvector $X_{1}$ for tornillo T1 and T2 estimated from the covariance matrix for moving $5 \mathrm{~s}$ intervals. The horizontal and vertical bars are the means and standard deviations, respectively.
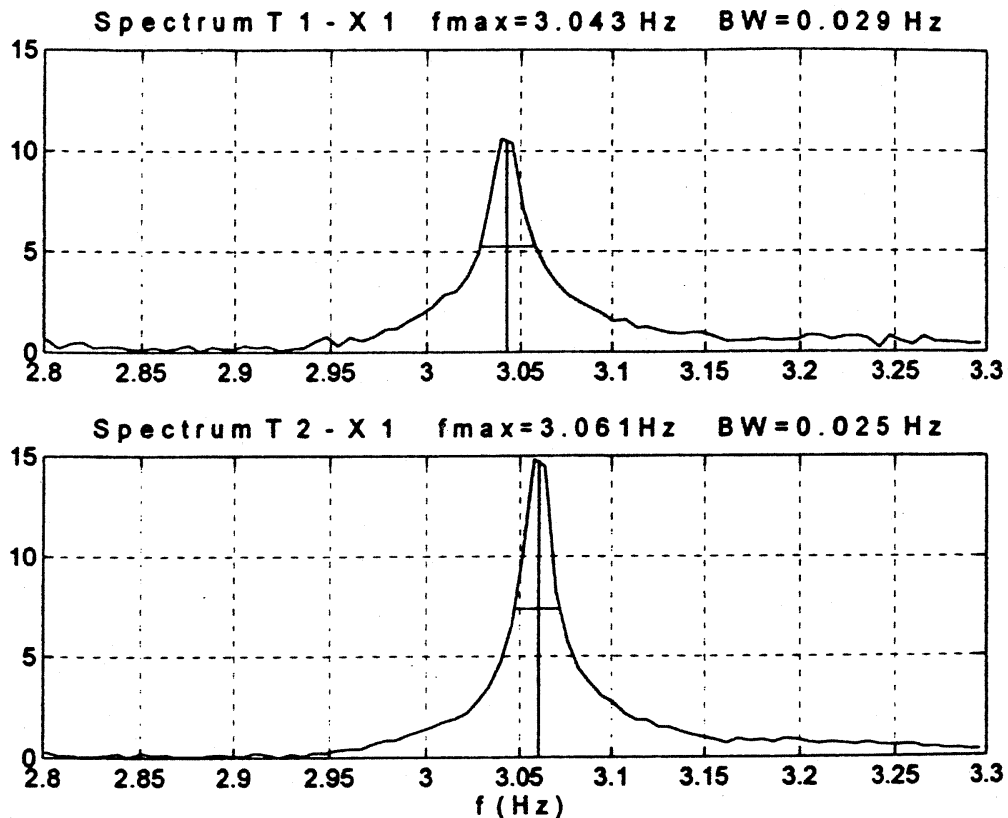

Fig. 7. Amplitude spectra: the amplitude spectra (units: $\mu \mathrm{m} / \mathrm{s} / \mathrm{Hz}$ ) for T1- $X_{1}$ and T2- $X_{1}$ in fig. 3 indicate small differences in the peak frequency $f_{\max }$ and in the $6 \mathrm{db}$ - bandwidths $B W$ for the two tornillos. 


\section{Spectra}

The tornillos T1 and T2 have peaked amplitude spectra with small differences in the peaks' frequencies, $f_{\max 1}=3.043 \mathrm{~Hz}$ and $f_{\max 2}=3.061$ $\mathrm{Hz}$, and bandwidths, $B W_{1}=0.029 \mathrm{~Hz}$ and $B W_{2}=0.025 \mathrm{~Hz}$, respectively (fig. 7). These spectra were calculated from a $90 \mathrm{~s}$ time series, so the frequency resolution is better than $\Delta f=0.01 \mathrm{~Hz}$. The frequency domain quality factors, which can be calculated from peaks' frequencies and bandwidths using the relationship $Q_{f}=f_{\max } /(B W / 2)$ (Leighton, 1994), are $Q_{f 1}=210$ and $Q_{f 2}=245$.

The time-frequency spectrogram for $\mathrm{T} 2-X_{1}$ in fig. 8 shows that the short-time average of the instantaneous peak frequencies is constant within the frequency resolution of $0.05 \mathrm{~Hz}$.

\section{Analytic function}

Extremely small variations in the frequency of amplitude- and frequency-modulated narrowband signals can be resolved to high precision from the analytic signal using the reduced instantaneous phase (Hellweg, 1999). Figure 9 shows the reduced instantaneous phase for tornillo T1 with a test frequency $f_{01}=3.0420 \mathrm{~Hz}$ and for T2 with $f_{02}=3.0595 \mathrm{~Hz}$. The instantaneous frequencies show small fluctuations but their short-term averages - defined by the slope of the least-square-fit - remain constant during the course of the tornillos, with less precise results for T1 due to this tornillo's lower signal-tonoise ratio. This is particularly obvious in the second half of the recording.

The amplitude envelope $a(t)$ of the analytic signal of the tornillos $\mathrm{T} 1$ and $\mathrm{T} 2$ is shown in fig. 10. At the beginning, the amplitude rises rather rapidly until it reaches a maximum. Most of the tornillo is characterized by a slow exponential decay. We can therefore consider the amplitude function of the tornillo, $a(t)$ (eq. (3.1)), to be made up of three parts:

1) An oscillator excitation function with increasing amplitude $e(t) \cdot e(t)$ can be approximated by the function $a_{e}\left(1-\exp \left(-q_{e} t\right)\right)$ with $q_{e} \approx 1$. The rise time $T_{R}$ of $e(t)$ is about $10 \mathrm{~s}$ for both tornillos.

2) A resonator response function with expo-
$\mathrm{T} 2-\mathrm{X} 1$
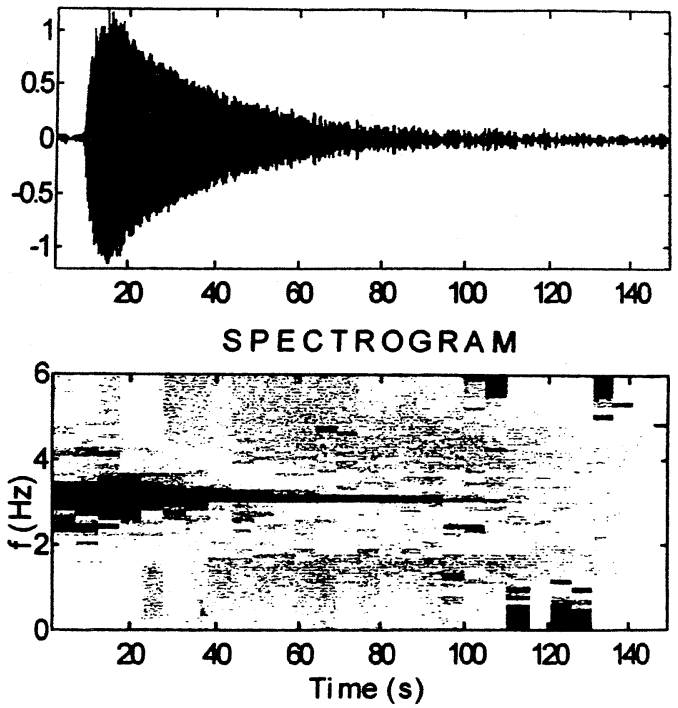

Fig. 8. Time-frequency spectrograms: timefrequency spectrograms for $\mathrm{T} 2-X_{1}$ indicating that the peak frequency is constant within the frequency resolution of $0.05 \mathrm{~Hz}$. Spectrogram estimation using Kaiser-windowed $5 \mathrm{~s}$ record segments with $50 \%$ overlapping.

nentially decaying amplitudes $a_{0} \exp \left(-q_{0} t\right) h\left(t-T_{R}\right)$. $h(t)$ is the unit step function.

3) A random function $b(t)$ describing the effects of scattering and seismic noise.

\section{Resonator impulse response}

In fig. 11 the amplitude spectrum of tornillo T2 is compared with the spectrum of the impulse response function of a linear resonator with the transfer function

$$
H(s)=\frac{1}{s^{2}+2 q_{0} s+\omega_{0}^{2}}
$$

where $q_{0}$ is the damping factor determined from the exponential decay of the tornillo envelope in fig. 10 and $\omega_{0}=2 \pi f_{0}$ is the angular frequency from the reduced instantaneous phase in fig. 9. 

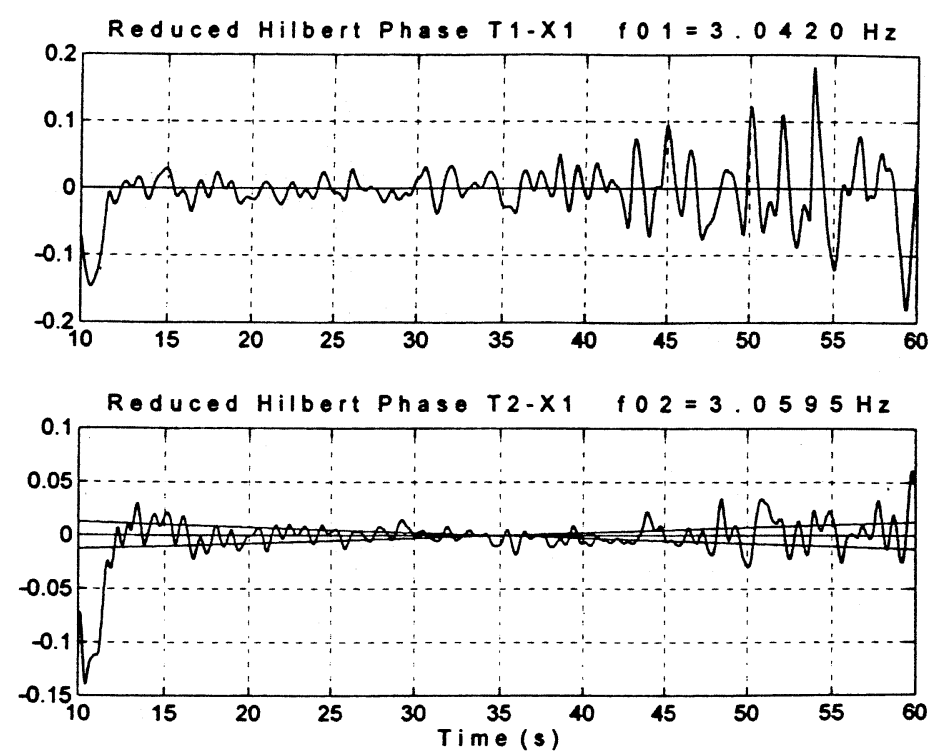

Fig. 9. Instantaneous frequency: reduced instantaneous phase from the analytic function $\left(r(t)=h(t) /(2 \pi)-f_{0} t\right.$ for T1 and T2. Changes of the instantaneous frequency $f_{\text {inst }}$ relative to the reference frequency $f_{0}$ occur when the slope, $f_{\text {inst }}=d r / d t+f_{0}$, changes. Thick solid line: $f_{\text {inst }}=f_{0}$. Thin solid lines: $f_{\text {inst }}=f_{0} \pm 0.0005$. The short-term average of the instantaneous frequency is extremely constant to better than $0.0002 \mathrm{~Hz}$.
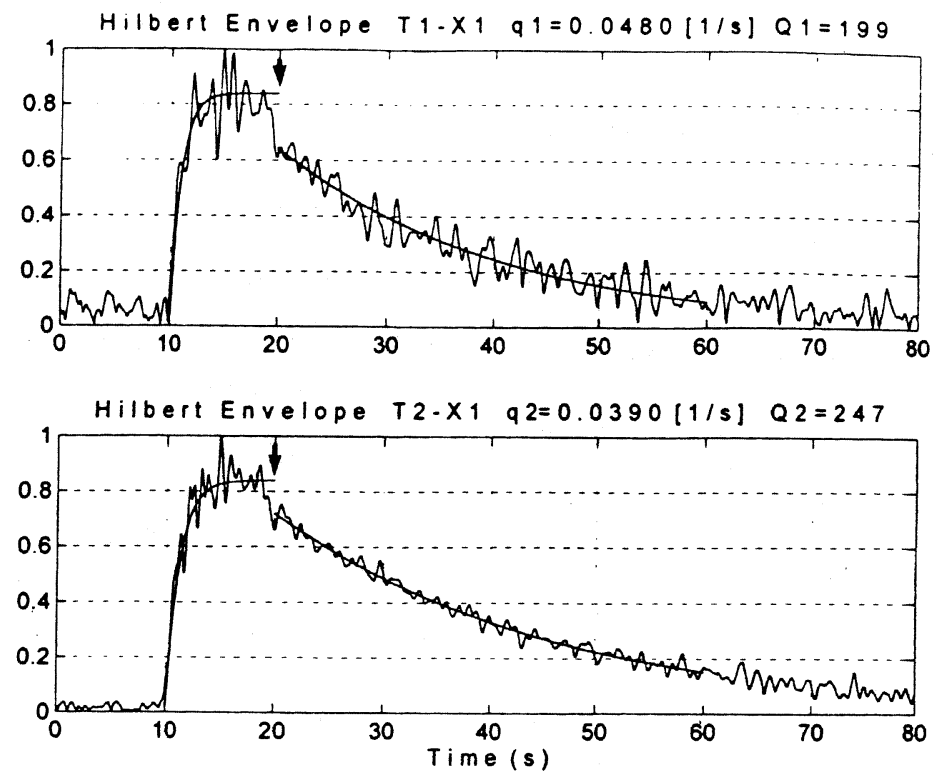

Fig. 10. Amplitude envelope: Hilbert amplitude envelope for T1- $X_{1}$ and $\mathrm{T} 2-X_{1}$ with two segments separated by the arrow about $10 \mathrm{~s}$ after the onset. Transient segment $(t<10 \mathrm{~s})$ : amplitudes increase with a rise time of about $10 \mathrm{~s}$ for both tornillos. Response segment $(t>10 \mathrm{~s})$ : amplitudes decay exponentially [ $\exp (-q t)]$. Solid line: least-square fit for the decay constant $q$. Time domain quality factor $Q=\pi f_{0} / q$, where $f_{0}$ is taken from fig. 9 . 


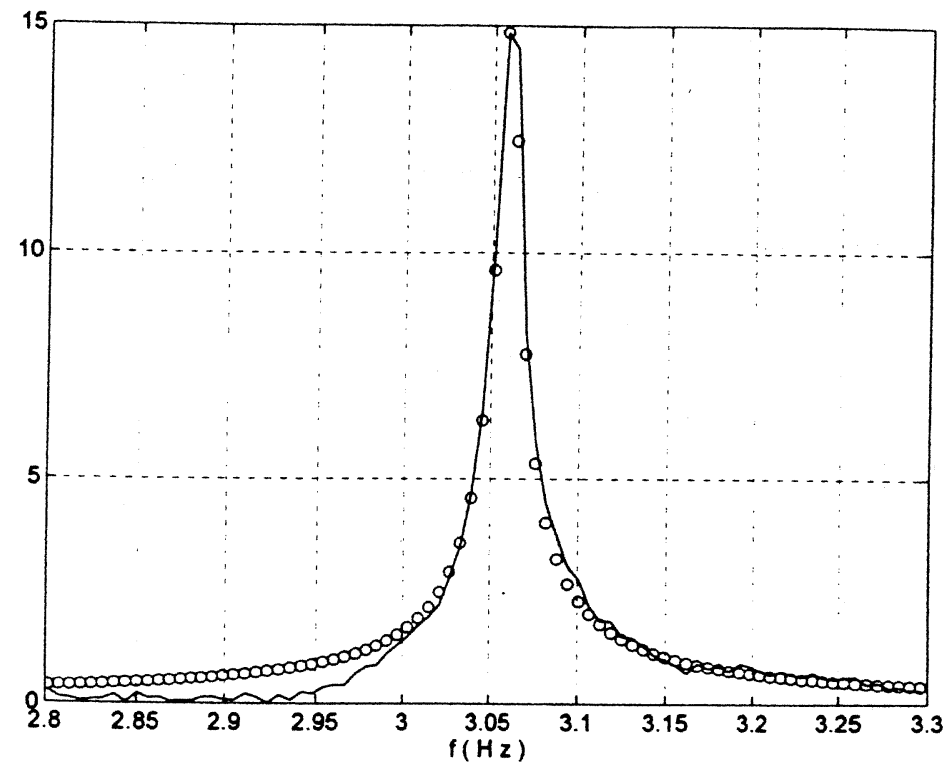

Fig. 11. Linear resonator source: the spectrum of tornillo $\mathrm{T} 2-X_{1}$ is compared with the impulse response of a resonator with transfer function given in eq. (7.1). Solid line: amplitude spectrum of tornillo T2- $X_{1}$. Circles: amplitude spectrum of the impulse response for a resonator with frequency $f_{0}=3.0595 \mathrm{~Hz}$ (fig. 7) and decay constant $q=h_{0} \omega_{0}=0.039$ (fig. 8 ), $\omega_{0}=2 \pi f_{0}$.

The discrepancy between the two functions between 2.8 and $3.0 \mathrm{~Hz}$ is caused by the finite rise time $T_{R}$ of the excitation function.

\section{Conclusions}

The observed close correlation between tornillos and ash eruptions invites the use of this type of seismovolcanic event as a parameter for characterizing the level of volcanic activity. It is therefore extremely important to develop methods to parametrize and classify tornillos. A detailed analysis of the time dependence of the variables in eq. (3.1) yields, on the one hand, a tool box for such an endeavour. It also lays the foundation for the development of a physical model of the processes in the volcano which generate the tornillo signals. Table I lists information about the excitation process, the coupling and the medium which can be obtained from such an analysis.

In the case of these two tornillos, several of these parameters, such as the predominant frequency, the polarization of the signals and the damping factor are extremely stable (see table II). These results together with the proposed separation of a tornillo into two distinct parts, an initial excitation followed by lightlydamped resonance (fig. 10) lead to the following conclusions about these tornillos:

- They are generated by a quasi-impulsive excitation process.

- The frequency of this process is the same as that of the resonator.

- The source and the resonator are highly stationary and are confined to a small volume.

- The polarization and spectral parameters of the resonator do not change measurably during a tornillo.

While these conclusions do yet not allow the formulation of a general source model for a tornillo, they nevertheless provide constraints on the physical parameters for source modelling. Progress on such a project can only be achieved, after more tornillos have been recorded with a denser network of three-component stations. 
Dieter Seidl, Margaret Hellweg, Horst Rademacher, Diego M. Gómez M. and Roberto A. Torres C.

Table I. Constraints for tornillo source modelling. The parameters from the general equation describing a tornillo (eq. (3.1)) may be related to the source process or may be effected by the medium.

\begin{tabular}{llll}
\hline \hline & Tornillo excitation process & \multicolumn{1}{c}{ Source of seismic wave } & \multicolumn{1}{c}{ Nearfield medium } \\
\hline$\vec{n}(t)$ & & Location, size, radiation pattern & Scattering \\
$\varphi(t)$ & Random or impulsive & & Scattering \\
$\omega(t)$ & Stationarity & Resonator & \\
$a(t)$ & Source-time function & Strength of coupling & Attenuation, scattering \\
\hline
\end{tabular}

Table II. Results of tornillo analysis. Characteristics of the parameters from the general equation describing a tornillo (eq. (3.1)) determined by analysis are listed along with their implications for a model of the tornillo source.

\begin{tabular}{ll}
\hline \hline$a(t)=a_{e}(t)+a_{0} e^{-q_{0} t}+b(t)$ & $\begin{array}{l}\text { The decay parameter, } \boldsymbol{q}_{0}, \text { is very small. The effect of scattering, } \boldsymbol{b}(\boldsymbol{t}), \\
\text { is random and small. }\end{array}$ \\
\hline$\omega(t)=\omega_{0}$ & The source of seismic waves is a single, weakly-damped resonator. \\
$q(t)=q_{0}$ & The excitation process is impulsive. \\
\hline$\varphi(t)=\varphi_{0}$ & $\begin{array}{l}\text { The tornillo stems from small volume source. The effects of scattering } \\
\text { are small. }\end{array}$ \\
\hline$\vec{n}(t)=\vec{n}_{0}$ &
\end{tabular}

\section{Acknowledgements}

The research on Galeras was performed as part of a joint project between the Federal Institute for Geosciences and Natural Resources (BGR, Germany) and the Instituto de Investigaciones en Geociencias Mineria y Quimica (INGEOMINAS, Colombia). Essential support was provided by the Forschungszentrum Jülich (Germany). We would like to thank Bruno Martinelli and the Swiss Embassy in Bogota for their helpful assistance in solving sophisticated customs problems.

\section{REFERENCES}

GómEZ, D. and R. TORRES (1997): Unusual low-frequency seismic events with slowly decaying coda wave observed at Galeras and other volcanoes, in Galeras Volcano, Colombia: Interdisciplinary Study of a Decade Volcano, edited by J. STIX, M.L. CALVACHE V. and S.N. WiLliams, J. Volcanol. Geotherm. Res., 77, 173-193.

GómEZ, M., D.M., R.A. TORRES C., D. SEIDL, M. HELlWEG and H. RADEMACHER (1999): Tornillo seismic events at Galeras volcano, Colombia: a summary and new information from broadband three-component measurements, Ann. Geofis., 42 (3), 365-378 (this volume).

HellWEG, M. (1999): Listening carefully: unique observations of harmonic tremor at Lascar volcano, Chile, Ann. Geofis., 42 (3), 451-464 (this volume).

KANASEWICH, E.R. (1981): Time Sequence Analysis in Geophysics (University of Alberta Press), 328-360.

LeIGHTON, T.G. (1994): The Acoustic Bubble (Academic Press, London), 172-173.

MARTINELli, B. (1991): Fluidinduzierte Mechanismen für die Entstehung von vulkanischen Tremor-Signalen, DISS.ETH No. 9376, Zürich.

Plešinger, A., M. HellweG and D. SeIdL (1986): Interactive high-resolution polarization analysis of broad-band seismograms, J. Geophysics, 59, 129-139.

SEIDL, D. and M. HELlWEG (1991): Volcanic tremor recordings: polarization analysis, in Volcanic Tremor and Magma Flow, edited by R. SCHICK and R. Mugiono, Germany, Kernforschungsanlage Jülich, $31-46$.

Torres, C., R.A., D.M. GómEZ M. and L.M. NARVAEZ (1996): Unusual seismic signals associated with the activity at Galeras volcano, Colombia, from July 1992 to September 1994, Ann. Geofis., 39 (2), 299-310.

WIELANDT, E. and G. STRECKEISEN (1982): The leaf-spring seismometer: design and performance, Bull. Seismol. Soc. Am., 72, 2349-2367. 